\title{
ABC Dementia Scale: A Quick Assessment Tool for Determining Alzheimer's Disease Severity
}

\author{
Takahiro Mori $^{a}$ Takashi Kikuchi ${ }^{b} \quad$ Yumi Umeda-Kameyamac \\ Kenji Wada-Isoe $^{d}$ Shinsuke Kojima ${ }^{b}$ Tatsuo Kagimurab

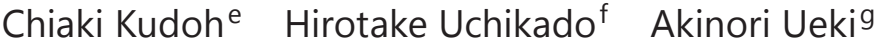 \\ Masato Yamashita ${ }^{h}$ Toshiyuki Watabe ${ }^{i}$ Chika Nishimuraj \\ Norifumi Tsuno ${ }^{a}$ Takashi Uedak $^{k}$ Masahiro Akishita ${ }^{c}$ \\ Yu Nakamura ${ }^{a}$ on behalf of the ABC Dementia Scale Study Group \\ ${ }^{a}$ Department of Neuropsychiatry, Kagawa University School of Medicine, Kagawa, Japan; \\ bTranslational Research Informatics Center, Foundation for Biomedical Research and \\ Innovation, Kobe, Japan; ' Department of Geriatric Medicine, Graduate School of Medicine, \\ The University of Tokyo, Tokyo, Japan; ${ }^{\mathrm{d}}$ Division of Neurology, Department of Brain and \\ Neurosciences, Faculty of Medicine, Tottori University, Yonago, Japan; ${ }^{\mathrm{K}} \mathrm{KUDOH}$ CHIAKI \\ Clinic for Neurosurgery and Neurology, Tokyo, Japan; ${ }^{f}$ Shonan Inaho Clinic, Hiratsuka, \\ Japan; ${ }^{9}$ Ueki Dementia and Geriatric Psychiatry Clinic, Nishinomiya, Japan; ${ }^{\text {hY }}$ amashita \\ Clinic, Neurosurgery and Rehabilitation, Ikoma, Japan; 'Watabe Clinic, Sakata, Japan; \\ ${ }^{j}$ Kurumi Clinic, Tokyo, Japan; kMedical Corporation Koujinkai, Ueda Neurosurgical Clinic, \\ Miyazaki, Japan
}

\section{Keywords}

Neuropsychological tests · Activities of daily living · Dementia $\cdot$ Cognition $\cdot$ Cognitive dysfunction

\section{Abstract}

Background: In this study, we examined the construct validity, concurrent validity concerning other standard scales, intrarater reliability, and changes in scores at 12 weeks of the previously developed ABC Dementia Scale (ABC-DS), a novel assessment tool for Alzheimer's dis-

Trial registration No. NCT02667665. Under the terms and conditions applied, any researcher can download the PDF files of the questionnaire of the ABC Dementia Scale and the evaluation guidance in English from the homepage of the Mapi Research Trust at http://mapi-trust.org/our-resources/questionnaires-distributed-by-the-mapi-research-trust/. Japanese researchers can obtain the Japanese version of the scale by sending a request e-mail to abc_scale@tri-kobe.org. 
ease (AD). Methods: Data were obtained from 312 patients diagnosed with either AD or mild cognitive impairment. The scores on the ABC-DS and standard scales were compared. $\boldsymbol{R e}$ sults: The 13 items of the $A B C$-DS are grouped into three domains, and the domain-level scores were highly correlated with the corresponding conventional scales. Statistically significant changes in assessment scores after 12 weeks were observed for the total ABC-DS scores. Conclusion: Our results demonstrate the ABC-DS to have good validity and reliability, and its usefulness in busy clinical settings.

(C) 2018 The Author(s)

Published by S. Karger AG, Basel

\section{Introduction}

While dementia patients are sometimes assessed using only cognitive function tests such as the Mini-Mental State Examination (MMSE) [1] and the revised version of Hasegawa's Dementia Scale (HDS-R) [2, 3], it has become increasingly important to evaluate other aspects of the disease, such as behavioral and psychological symptoms of dementia (BPSD), typically measured using the Neuropsychiatric Inventory (NPI) [4], and activities of daily living (ADL) function, often measured using the Disability Assessment for Dementia (DAD) [5]. However, conventional neuropsychological assessments are designed to target either BPSD or ADL function, not both, and for a long time, few instruments were available to measure both aspects together with cognitive function in an integrated way [6]. Moreover, evaluators must receive professional training to be able to administer tests like the NPI and the Clinical Dementia Rating (CDR) [7] properly.

The CDR uses a 5-point rating system to assess dementia on six domains: memory, orientation, judgment and problem-solving, community affairs, home and hobbies, and personal care. An algorithm is used to combine the individual ratings in each of the six domains to obtain a Global CDR score, where a CDR score of 0 indicates normal cognition and CDR scores of $0.5,1,2$, and 3 indicate questionable/very mild, mild, moderate, and severe dementia, respectively. Despite its high reliability and continued use for over 30 years throughout the world, the scale has not been used much in daily clinical practice in Japan.

Therefore, the field requires an easy-to-use scale for diagnosing Alzheimer's disease (AD) which can be employed not only by dementia specialists but also by nonspecialists. One proposed solution is the Relevant Outcome Scale for Alzheimer's Disease (ROSA) [8]. Developed in Germany, this 16-item, 21-point instrument can comprehensively assess a patient's cognitive function, ADL function, BPSD, communication ability, and quality of life in approximately $15 \mathrm{~min}$. However, the ROSA requires changing the scenario according to stage (i.e., for early-, middle-, and later-stage AD). Therefore, its administration requires the evaluator first to establish the severity of a patient's AD using other objective indices, thereby complicating its use in clinical practice. The requirement of training makes the tool even more cumbersome, and training-related materials do not provide clear guidance on how to interpret the nonintuitive scores (e.g., 8.5 points) possible on this 21-point scale. For these reasons, the authors formed a study group to develop a dementia rating scale that can overcome the limitations of the ROSA.

The development of the ABC Dementia Scale (ABC-DS) involved a 3-step process from 2014 to 2016. In step 1, dementia specialists developed the questions and responses for the 17 items. In step 2, the construct validity, subjective group discriminability, and interrater reliability of the question items were examined using data from 545 patients (manuscript in preparation). These revisions resulted in a new, 13-item version of the ABC-DS. The details of this development will be reported in another paper. In this paper, we report its concurrent validity with other standard dementia assessment scales (i.e., the MMSE, NPI Caregiver 
8. How is the patient when something goes against his/her wishes?
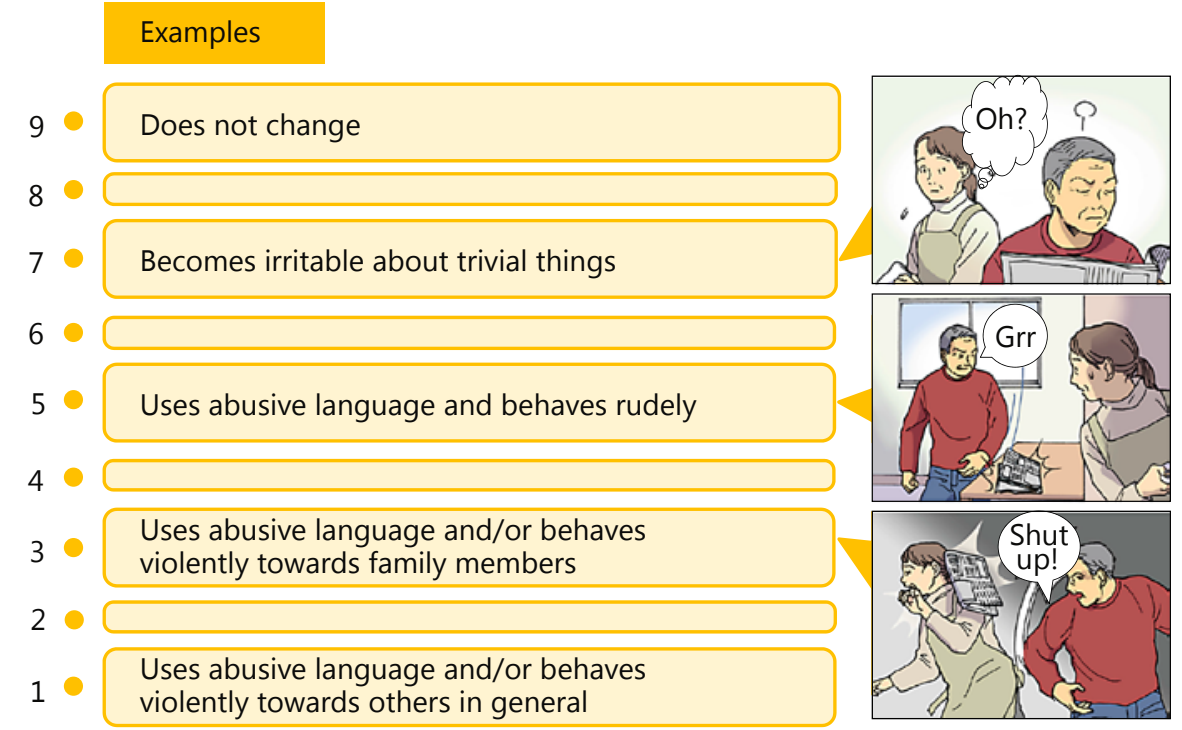

Fig. 1. A sample question of the $A B C$ Dementia Scale to evaluate behavioral and psychological symptoms of dementia.

Distress Scale [NPI-D], DAD, and CDR), intrarater reliability, and responsiveness to change over a 12-week interval and discuss its utility for a quick diagnosis in medical practice using the total score.

\section{Subjects and Methods}

\section{Participants}

Participants were patients examined at 22 clinics and hospitals across Japan who had been diagnosed with (1) AD based on the criteria of the Diagnostic and Statistical Manual of Mental Disorders, 4th edition, text revision (DSM-IV-TR) [9], (2) probable AD based on the criteria of either the National Institute on Aging-Alzheimer's Association (NIA-AA) workgroups [10] or the National Institute of Neurological and Communicative Disorders and Stroke and the Alzheimer's Disease and Related Disorders Association [11], or (3) mild cognitive impairment (MCI) based on the DSM-IV-TR or NIA-AA diagnostic criteria [12]. To be included in the study, patients needed to be receiving assistance from a caregiver 3 or more days a week, or cohabiting with them. Individuals with forms of dementia besides AD were excluded, as were those with comorbid major neurological or mental disorders.

\section{ABC Dementia Scale}

The ABC-DS uses a 9-point scale for each question item, with lower scores indicating poorer function (Fig. 1). Evaluators question caregivers about their patient with a semi-structured interview. Anchor points are provided for points 1, 3, 5, 7, and 9, with typical symptoms defined 
in sentences. Raters can also select intermediate responses (i.e., points 2, 4, 6, and 8) for cases that seem to match two adjacent anchor points. For example, if a patient's condition equals both points 7 and 9, the evaluator selects point 8 . The intermediate responses (i.e., points 2, 4, 6, and 8) do not contain scale descriptions in writing. Additionally, we added illustrations for points 3 , 5 , and 7 to make it easier for caregivers to associate them with their patient's symptoms. The total ABC-DS score is the arithmetic sum of the scores of the 13 items of the ABC-DS, and ranges from 13 to 117. A license for the ABC-DS can be purchased from the Mapi Research Trust (http:// mapi-trust.org/our-resources/questionnaires-distributed-by-the-mapi-research-trust/).

\section{Instruments}

In the present study, the ABC-DS was assessed for construct and concurrent validity, test and item information, intrarater reliability, and responsiveness to change. Several instruments were used to confirm the construct and concurrent validities of the ABC-DS. The patients were assessed by health professionals using the ABC-DS: $13.5 \%$ by doctors, $41.7 \%$ by nurses, $3.5 \%$ by clinical psychologists, and $41.3 \%$ by other kinds of healthcare workers. Cognitive function was assessed using the MMSE, BPSD with the NPI-D, and ADL function with the DAD. The patients were also assessed using the CDR, regarding both Global CDR and CDR Sum-of-Boxes (CDR-SB) scores. These standard dementia assessments were conducted according to their corresponding official manuals by a doctor or clinical psychologist other than the person administering the ABC-DS. The ABC-DS evaluator did not share his/her results with the person who conducted the standard dementia assessments, and vice versa.

\section{Construct Validity}

The ABC-DS item-level scores were subjected to factor analysis with oblique promax rotation to calculate factor loadings. The number of factors was identified, along with their constituent items, and their cumulative contribution rate was calculated.

\section{Qualitative Assessment of the Item Contents Based on Item Response Theory}

Item response theory is a statistical approach to develop survey instruments with high reliability [13]. This theory is typically used for sociological and psychological surveys, and the number of reports on its use in the development of assessment tools for dementia has been increasing since the 2000s [14-16].

We applied a graded response model for each domain separately, assuming "unidimensionality within a domain," whereby the items would measure a single common trait of ADL, BPSD, or cognitive function. We evaluated the quality of the ABC-DS items utilizing the item response category characteristic curve (IRCCC), a technique known in item response theory [17]. In this method, each possible response ("level") for an item is graphed as a probability density curve to depict its likelihood of being chosen. In our case, the levels are a continuous variable that reflects statistically converted $\mathrm{AD}$ severity, and by noting how the severity changes, we can evaluate changes in the likelihood of each level being chosen.

To interpret the IRCCCs, we checked the following points: (1) the curves must be reasonably located, reflecting the order of the ordered categorical scale; (2) the difficulty parameter must be located between -4 and 4 ; (3) the locations of the curves must maintain a sufficient distance from each other; and (4) the steepness of the curves must be sufficient, while the discrimination parameter must be $>0.2$ and $<4.0$.

We used the R package Itm to analyze the IRCCCs [18]. However, as this package cannot handle 9-level items, the responses were adapted to 5 modified levels: level 1 for 1-2 points, level 2 for 3-4 points, level 3 for 5 points, level 4 for 6-7 points, and level 5 for 8-9 points. Each item was thus analyzed by the parameters describing the 5 probability density curves corresponding to the 5 levels, acknowledging loss of information. 


\section{Internal Consistency}

The internal consistency of the ABC-DS was evaluated using Cronbach's $\alpha$ and $\omega$ reliability coefficients.

\section{Concurrent Validity}

Concurrent validity was assessed regarding correlations of the total ABC-DS score and each domain-level score with MMSE, NPI-D (both symptom scores and caregiver distress scores), DAD, CDR-SB, and Global CDR scores. Correlations with Global CDR scores were evaluated using polyserial correlation coefficients; Pearson's correlation coefficient was used for all other correlations.

\section{Intrarater Reliability}

The ABC-DS was re-administered to 218 participants who visited their clinics or hospitals 1 week after the initial assessment, by the same evaluator who carried out the first assessment. Weighted $\kappa$ coefficients and 95\% confidence intervals (CI) were calculated for the individual items. Intraclass correlation coefficients and 95\% CI were calculated for the total ABC-DS score.

\section{Predictive Ability of the Global CDR Score Using Receiver Operating Characteristic}

Curve Analysis

We performed receiver operating characteristic (ROC) curve analysis to investigate the sensitivity and specificity of the total ABC-DS score for discriminating the severity of AD defined by the Global CDR. We identified the most appropriate thresholds for the total ABC-DS score to discriminate $\mathrm{AD}$ severity using the R 3.1.0 statistical software with the ROCR package [18].

The sensitivities and specificities at the thresholds were for CDR $0 / 0.5$ versus $1 / 2 / 3$, CDR $0 / 0.5 / 1$ versus $2 / 3$, and CDR $0 / 0.5 / 1 / 2$ versus 3 . In this analysis, we defined that the test is positive (indicating a better stage) if the total ABC-DS scores were above the threshold. The sensitivity and specificity were described as $P$ (positive|positive) and $P$ (negative|negative), respectively, where $P(X \mid X)$ indicates the probability of a correct discrimination that the test diagnosed is $X$ when the patient is truly at $X ; X$ could be negative or positive.

\section{Twelve-Week Changes in Assessment Scores}

The ABC-DS, DAD, NPI-D, MMSE, and CDR were re-administered to 227 participants who visited their clinics or hospitals 12 weeks after the initial examination. Differences between the scores on the initial test and those on the 12-week test were calculated, along with $95 \%$ CI. Moreover, coefficients of variation (standard deviation/mean) were calculated for each measure.

\section{Statistical Analysis}

SAS version 9.3 (SAS Institute Inc., Cary, NC, USA) and R version 3.1.9 were used for all statistical analyses.

\section{Results}

\section{Patients}

Initially, 327 participants were enrolled in the study. However, 3 of them were excluded after registration for being deemed ineligible to participate, while another 12 were excluded for being assessed with tests not stipulated in the study protocol. The analysis population 
Table 1. Results of the factor analysis and intrarater reliability assessment of the ABC-DS

\begin{tabular}{|c|c|c|c|c|}
\hline \multirow[t]{2}{*}{ Item } & \multirow[t]{2}{*}{ Question } & \multicolumn{2}{|c|}{ Factor analysis } & \multirow{2}{*}{$\begin{array}{l}\begin{array}{l}\text { Intrarater } \\
\text { reliability }\end{array} \\
\begin{array}{l}\text { weighted } \\
\text { coefficient }\end{array}\end{array}$} \\
\hline & & domain & $\begin{array}{l}\text { factor } \\
\text { loading }\end{array}$ & \\
\hline Q1 & Daily activities: How is the patient when changing clothes? & A & 0.884 & 0.801 \\
\hline Q2 & $\begin{array}{l}\text { Motivation: How spontaneously does the patient perform the activities of } \\
\text { daily living? }\end{array}$ & A & 0.581 & 0.653 \\
\hline Q3 & $\begin{array}{l}\text { Communication: When the patient wants to communicate something, how } \\
\text { much is he or she able to do so through conversation? }\end{array}$ & A & 0.714 & 0.659 \\
\hline Q4 & $\begin{array}{l}\text { Complex acts: How is the patient when using home appliances such as the } \\
\text { television or air conditioner? }\end{array}$ & A & 0.606 & 0.749 \\
\hline Q5 & $\begin{array}{l}\text { Recent event memory: How well can the patient remember the location of a } \\
\text { familiar item? }\end{array}$ & $\mathrm{C}$ & 0.669 & 0.665 \\
\hline Q6 & $\begin{array}{l}\text { Recent event memory: How long does the patient remember the day-to-day } \\
\text { events taking place around him or her? }\end{array}$ & $\mathrm{C}$ & 0.930 & 0.738 \\
\hline Q7 & Restlessness: How is the patient when required to sit quietly? & $\mathrm{B}$ & 0.496 & 0.701 \\
\hline Q8 & Irritability: How is the patient when something goes against his/her wishes? & $\mathrm{B}$ & 0.744 & 0.594 \\
\hline Q9 & Cooperativeness: How is the patient when asked to do something? & B & 0.785 & 0.525 \\
\hline Q10 & Medication: How much help does the patient need to take medication? & $\mathrm{C}$ & 0.774 & 0.783 \\
\hline Q11 & Meals: How much help does the patient need to have a meal? & A & 0.814 & 0.780 \\
\hline Q12 & Toilets: How much help does the patient need to use the bathroom? & A & 0.965 & 0.809 \\
\hline Q13 & Care-burden: How much do you need to supervise the patient? & $\mathrm{C}$ & 0.541 & 0.775 \\
\hline
\end{tabular}

Domain: A, related to activities of daily living function; B, related to behavioral and psychological symptoms of dementia; C, related to cognitive function. ABC-DS, ABC Dementia Scale.

thus included 312 participants, of which 63 had MCI (20.2\%), 88 had mild AD (28.2\%), 106 had moderate AD (34.0\%), and 55 had severe AD (17.6\%). The population included 126 males (40.4\%) and 186 females (59.6\%) and had an average age of $80.6 \pm 7.1$ years (mean \pm SD). The patients had completed $11.11 \pm 2.61$ years of education (note: compulsory education lasts 9 years in the Japanese school system.). The patients' scores for MCI on the initial evaluation were as follows: DAD, $91.61 \pm 16.76$; NPI-D, $6.7 \pm 9.5$; MMSE, $25.8 \pm 3$; and CDR-SB, $2.33 \pm 2.01$. The patients' scores for mild $\mathrm{AD}$ on the initial evaluation were as follows: $\mathrm{DAD}$, $74.33 \pm 22.97$; NPI-D, $8.2 \pm 9.7$; MMSE, $21.6 \pm 2.9$; and CDR-SB, $5.07 \pm 2.32$. The patients' scores for moderate AD on the initial evaluation were as follows: DAD, $63.46 \pm 20.82$; NPI-D, $11 \pm 11$; MMSE, $16.9 \pm 3.8$; and CDR-SB, $8.08 \pm 3.33$. The patients' scores for severe AD on the initial evaluation were as follows: DAD, $34.54 \pm 27.6$; NPI-D, $21 \pm 17.5$; MMSE, $6.9 \pm 6$; and CDR-SB, $14.46 \pm 3.67$.

\section{Duration of Test Administration}

The average amount of time required to perform each assessment was as follows: ABC-DS, $9.96 \pm 4.79 \mathrm{~min}$; MMSE, $9.77 \pm 3.14 \mathrm{~min}$; NPI-D, $15.37 \pm 8.07 \mathrm{~min}$; DAD, $11.43 \pm 5.21 \mathrm{~min}$; and CDR, $26.39 \pm 9.84 \mathrm{~min}$.

\section{Construct Validity}

Factor analysis grouped the 13 items of the ABC-DS into three domains containing highly intercorrelated items: domain A, related to ADL function; domain B, related to BPSD; and domain $\mathrm{C}$, related to cognitive function (Table 1 ). These three factors had a cumulative contribution rate of 0.585 . 


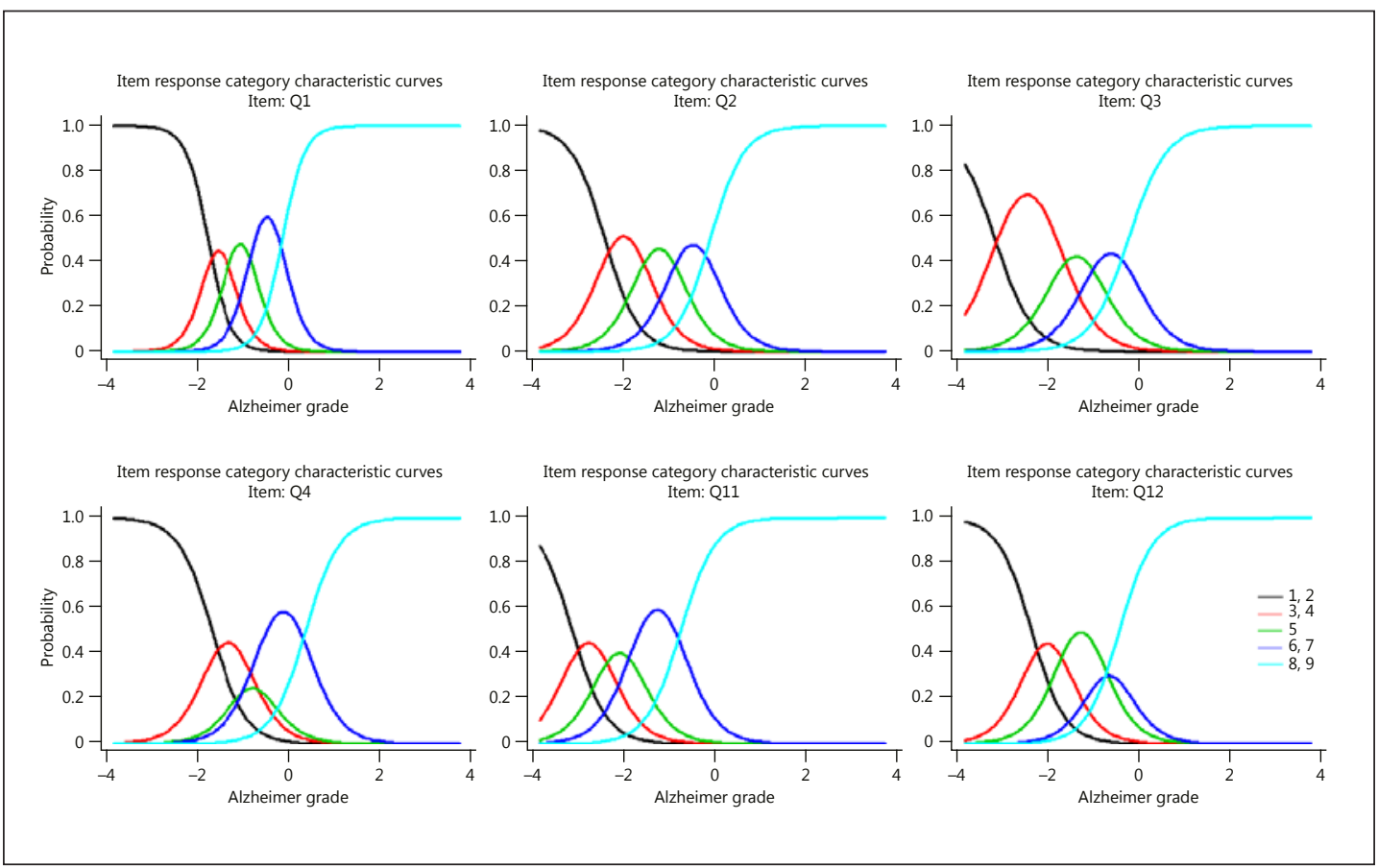

Fig. 2. Item response category characteristic curves for domain $A$ of the $A B C$ Dementia Scale.

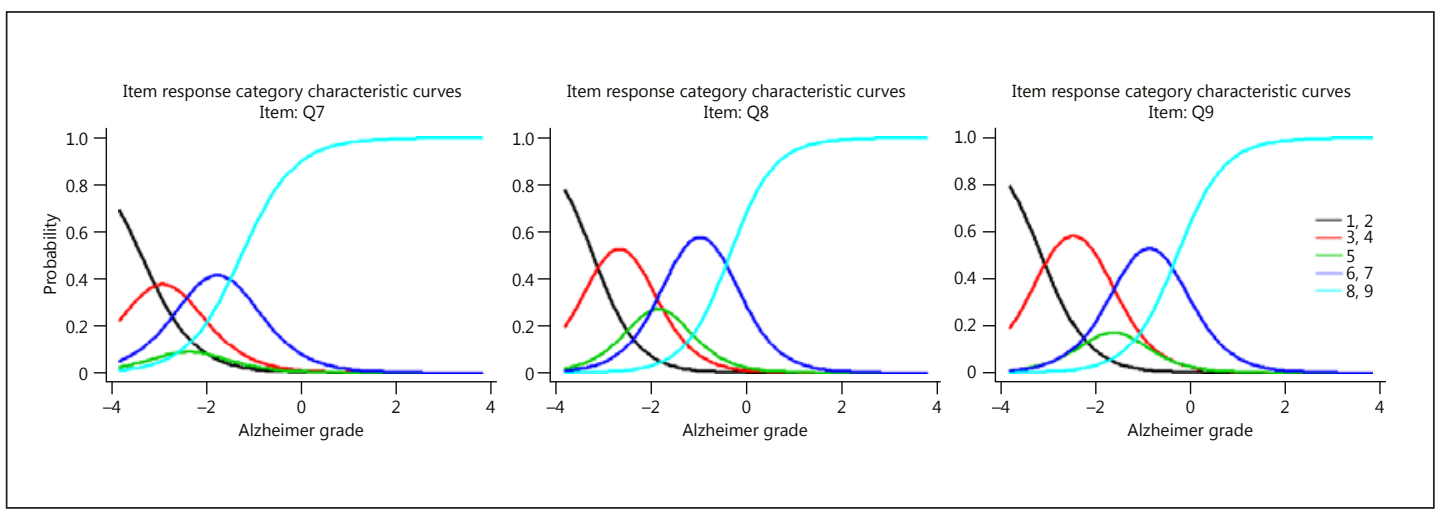

Fig. 3. Item response category characteristic curves for domain B of the ABC Dementia Scale.

We also checked the four-factor and five-factor models. The four-factor model did not have any belonging item, with the largest factor loading at the fourth domain. The five-factor model contained only one belonging item in the fourth and fifth domain each. These findings are consistent with those in step 2 (data not shown). Accordingly, we concluded that having more than three factors was redundant.

\section{Qualitative Assessment of the Items Based on Item Response Theory}

The IRCCCs for the items in domains A-C are shown in Figures 2-4, respectively. The curves for each modified level are colored as follows: level 1, black; level 2, red; level 3, green; level 4, blue; and level 5, light blue. In each domain, all items satisfied the preset criteria for the difficulty and discrimination parameters (Table 2). 


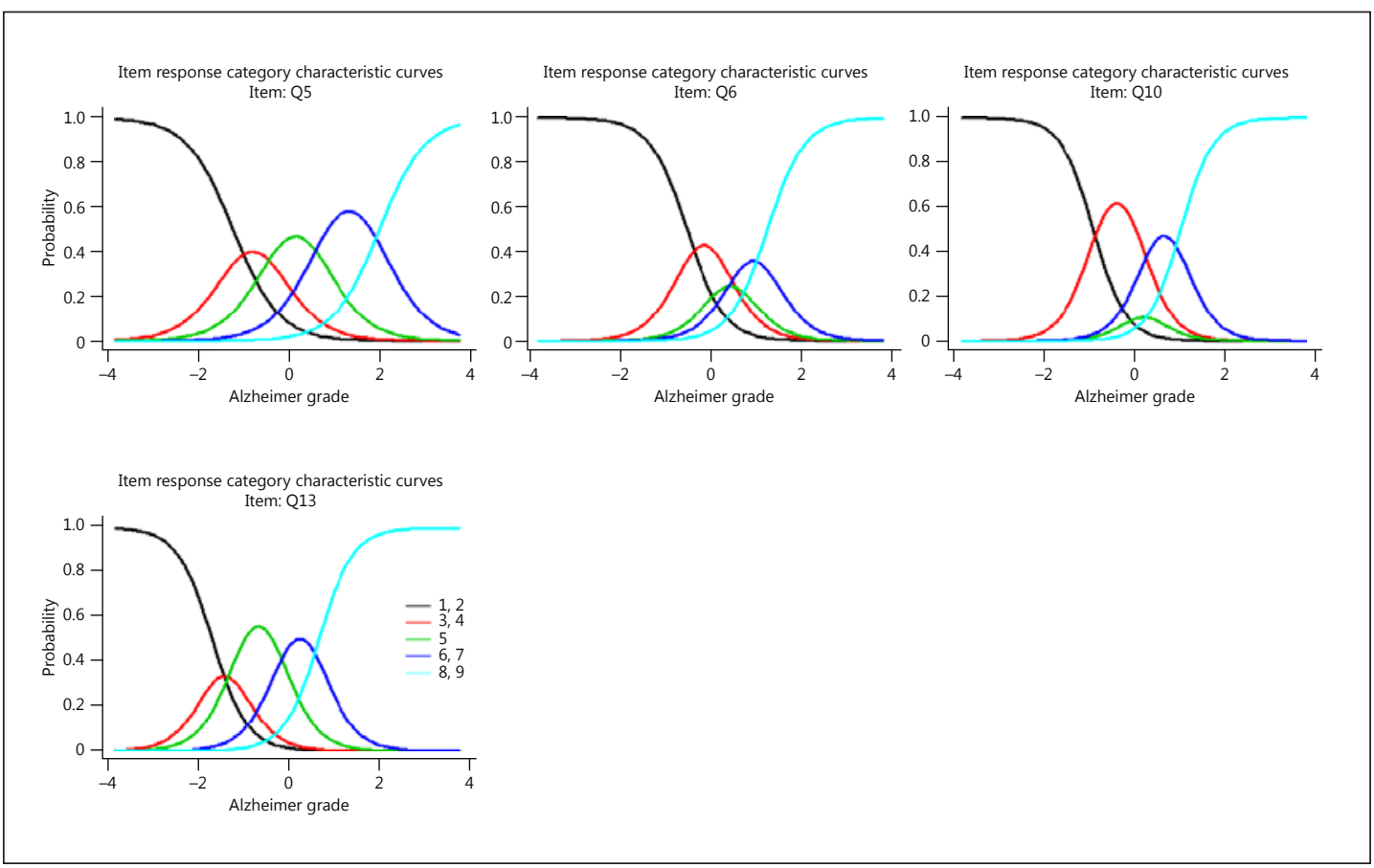

Fig. 4. Item response category characteristic curves for domain $\mathrm{C}$ of the $\mathrm{ABC}$ Dementia Scale.

\section{Internal Consistency}

The ABC-DS had acceptable internal reliability $(\alpha=0.915, \omega=0.921)$.

\section{Concurrent Validity}

Domain A scores were found to correlate with DAD scores, which also reflect ADL function $(r=0.674)$. Domain B scores were found to correlate with NPI-D scores, which also reflect BPSD $(r=-0.644)$. Domain C scores were found to correlate with MMSE scores, which also reflect cognitive function $(r=0.698)$.

Total ABC-DS scores correlated with other measures as follows: MMSE scores $(r=0.747)$, NPI-D Symptom scores ( $r=-0.595)$, NPI-D Caregiver Distress scores $(r=-0.540)$, DAD scores $(r=0.720)$, CDR-SB scores $(r=-0.840)$, and Global CDR scores $(r=-0.828)$ (Table 3$)$. These findings demonstrate strong correlations (i.e., $|r|>0.6$ ) of total ABC-DS scores with scores on all the conventional rating scales examined, except for the NPI-D Symptom and Caregiver Distress scores. Particularly strong correlations were exhibited with CDR-SB and Global CDR scores.

\section{Intrarater Reliability}

Intrarater reliability was confirmed by comparing scores assessed by the same evaluator on two occasions, a week apart. The weighted $\kappa$ coefficients were $>0.6$ for all items except for Q8 and Q9 in domain 3 (Table 1). The total ABC-DS scores had an intraclass correlation coefficient of 0.964 (95\% CI: 0.954-0.972).

\section{Predictive Ability of the Global CDR Score Using ROC Curve Analysis}

Cutoff values for predicting Global CDR scores based on total ABC-DS scores were established using ROC curve analysis (Table 4). The following thresholds were calculated for discriminating between Global CDR levels: CDR 0/0.5 versus 1/2/3, ABC-DS threshold = 
Table 2. Parameters of the IRCCCs

\begin{tabular}{|c|c|c|c|c|c|c|c|}
\hline & \multicolumn{5}{|c|}{ Difficulty $^{1}$} & \multirow{2}{*}{$\begin{array}{l}\text { Discrimi- } \\
\text { nation }\end{array}$} & \multirow{2}{*}{$\begin{array}{l}\text { Item } \\
\text { information }\end{array}$} \\
\hline & black & red & green & blue & light blue & & \\
\hline Q1 & -1.741 & -1.507 & -1.019 & -0.430 & -0.094 & 4.099 & 11.940 \\
\hline Q2 & -2.366 & -1.951 & -1.175 & -0.434 & -0.054 & 2.716 & 7.680 \\
\hline Q3 & -3.152 & -2.446 & -1.367 & -0.610 & -0.224 & 2.425 & 7.105 \\
\hline Q4 & -1.649 & -1.294 & -0.754 & -0.075 & 0.418 & 2.712 & 7.103 \\
\hline Q11 & -3.076 & -2.717 & -2.040 & -1.211 & -0.700 & 2.670 & 7.525 \\
\hline Q12 & -2.348 & -2.003 & -1.266 & -0.649 & -0.424 & 2.756 & 7.120 \\
\hline Q7 & -3.351 & -2.901 & -2.350 & -1.747 & -1.244 & 1.765 & 3.780 \\
\hline Q8 & -3.219 & -2.667 & -1.850 & -0.966 & -0.346 & 2.120 & 5.800 \\
\hline Q9 & -3.139 & -2.480 & -1.647 & -0.887 & -0.302 & 2.017 & 5.274 \\
\hline Q5 & -1.201 & -0.771 & 0.175 & 1.363 & 2.036 & 1.974 & 5.594 \\
\hline Q6 & -0.530 & -0.154 & 0.430 & 0.944 & 1.252 & 2.434 & 5.692 \\
\hline Q10 & -0.899 & -0.382 & 0.212 & 0.657 & 1.026 & 2.783 & 6.949 \\
\hline Q13 & -1.659 & -1.391 & -0.640 & 0.267 & 0.691 & 2.602 & 7.196 \\
\hline
\end{tabular}

IRCCC, item response category characteristic curve. ${ }^{1}$ Color coded. The color of the IRCCC for each item is shown in Figures 2-4.

Table 3. Correlation of total ABC-DS scores with scores on reference scales

\begin{tabular}{lccc}
\hline & $\begin{array}{l}\text { Correlation } \\
\text { coefficient }\end{array}$ & $\begin{array}{l}\text { Lower band of the } \\
95 \% \text { confidence interval }\end{array}$ & $\begin{array}{l}\text { Upper band of the } \\
\text { 95\% confidence interval }\end{array}$ \\
\hline MMSE & 0.747 & 0.693 & 0.792 \\
NPI-D (Symptoms) & -0.595 & -0.662 & -0.518 \\
NPI-D (Caregiver Distress) & -0.540 & -0.615 & -0.456 \\
DAD & 0.720 & 0.662 & 0.770 \\
CDR-SB & -0.840 & -0.870 & -0.804 \\
Global CDR & -0.828 & -0.864 & -0.792 \\
\hline
\end{tabular}

ABC-DS, ABC Dementia Scale; total ABC-DS score, sum of the scores of the 13 items of the ABC-DS; MMSE, Mini-Mental State Examination; NPI-D, Neuropsychiatric Inventory - Caregiver Distress Scale; DAD, Disability Assessment for Dementia; CDR-SB, Clinical Dementia Rating Sum-of-Boxes; CDR, Clinical Dementia Rating.

Table 4. Discrimination of Global CDR score by total ABC-DS score

\begin{tabular}{|c|c|c|c|c|c|}
\hline \multicolumn{2}{|l|}{ Comparisons: A vs. B } & \multirow[t]{2}{*}{ Threshold } & \multirow{2}{*}{$\begin{array}{l}\text { Sensitivity } \\
P(A \mid A)\end{array}$} & \multirow{2}{*}{$\begin{array}{l}\text { Specificity } \\
P(B \mid B)\end{array}$} & \multirow[t]{2}{*}{ AUC } \\
\hline A & B & & & & \\
\hline CDR $0 / 0.5$ & CDR 1 , CDR 2, and CDR 3 & 100.00 & 0.68 & 0.92 & 0.89 \\
\hline CDR $0 / 0.5$ and CDR 1 & CDR 2 and CDR 3 & 85.50 & 0.79 & 0.80 & 0.88 \\
\hline CDR $0 / 0.5$, CDR 1 , and CDR 2 & CDR 3 & 70.50 & 0.89 & 0.97 & 0.97 \\
\hline
\end{tabular}

If the total $\mathrm{ABC}$-DS score is higher than the threshold, it is defined to be lower in severity. ABC-DS, ABC Dementia Scale; total ABC-DS score, sum of the scores of the 13 items of the ABC-DS; CDR, Clinical Dementia Rating; AUC, area under the receiver operating characteristic curve. 
Table 5. Estimation of Global CDR score by total ABC-DS score

\begin{tabular}{ll}
\hline Total ABC-DS score range & Estimated Global CDR score \\
\hline $117-101$ & $0 / 0.5$ \\
$100-86$ & 1 \\
$85-71$ & 2 \\
$70-13$ & 3 \\
\hline
\end{tabular}

The left column shows the ranges of the total ABC-DS score to estimate the Global CDR score. ABC-DS, ABC Dementia Scale; total ABC-DS score, sum of the scores of the 13 items of the ABC-DS; CDR, Clinical Dementia Rating.

Table 6. Changes in scores at 12 weeks

\begin{tabular}{llrrrrrc}
\hline Scale & Subjects, $n$ & Mean & Lower & Upper & SE & CV & $p$ value \\
\hline ABC-DS domain A & 223 & -0.99 & -1.43 & -0.54 & 3.40 & -3.44 & $<0.0001$ \\
ABC-DS domain B & 223 & 0.16 & -0.17 & 0.50 & 2.55 & 15.77 & 0.345 \\
ABC-DS domain C & 223 & -0.89 & -1.35 & -0.43 & 3.50 & -3.92 & 0.000 \\
Total ABC-DS score & 223 & -1.72 & -2.52 & -0.91 & 6.12 & -3.56 & $<0.0001$ \\
DAD & 218 & -4.77 & -6.53 & -3.01 & 13.22 & -2.77 & $<0.0001$ \\
MMSE & 223 & -0.35 & -0.68 & -0.01 & 2.51 & -7.28 & 0.041 \\
NPI-D & 223 & 0.13 & -0.98 & 1.25 & 8.47 & 62.97 & 0.813 \\
CDR-SB & 223 & 0.33 & 0.18 & 0.49 & 1.19 & 3.57 & $<0.0001$ \\
\hline
\end{tabular}

ABC-DS, ABC Dementia Scale; total ABC-DS score, sum of the scores of the 13 items of the ABC-DS; DAD, Disability Assessment for Dementia; MMSE, Mini-Mental State Examination; NPI-D, Neuropsychiatric Inventory - Caregiver Distress Scale; CDR-SB, Clinical Dementia Rating Sum-of-Boxes; Lower, lower band of the $95 \%$ confidence interval; Upper, upper band of the 95\% confidence interval; SE, standard error; CV, coefficient of variation (i.e., standard deviation/mean).

100.0 points (sensitivity 0.68 , specificity 0.92 ); CDR $0 / 0.5 / 1$ versus $2 / 3$, ABC-DS threshold = 85.5 points (sensitivity 0.79 , specificity 0.80 ); and CDR $0 / 0.5 / 1 / 2$ versus 3 , ABC-DS threshold $=70.5$ points (sensitivity 0.89 , specificity 0.97 ). The area under the receiver operating curve for CDR $0 / 0.5$ versus $1 / 2 / 3$ was 0.89 , that for CDR $0 / 0.5 / 1$ versus $2 / 3$ was 0.88 , and that for CDR $0 / 0.5 / 1 / 2$ versus 3 was 0.97 . We developed a simple chart for estimating Global CDR scores based on total ABC-DS scores (Table 5).

\section{Twelve-Week Changes in Assessment Scores}

Table 6 shows the change in scores for each of the assessments 12 weeks after the initial evaluation. All measures except for ABC-DS domain B and NPI-D exhibited statistically significant changes at 12 weeks $(p<0.05)$. The absolute coefficient of variation of the total ABC-DS scores was lower than that of the CDR-SB scores, indicating that the ABC-DS possesses higher measurement repeatability and reliability.

\section{Discussion}

This article describes the reliability and validity of the ABC-DS. Concurrent validity was established in relation to several conventional dementia scales (DAD, MMSE, NPI-D, and CDR). Furthermore, the ABC-DS domain-level scores were highly correlated (i.e., $|r|>0.6$ ) 
with the scores on the standard scales corresponding to the same dimensions of AD: domain A scores, which reflect ADL function, correlated with DAD scores; domain B scores, reflecting BPSD, correlated with NPI-D scores; and domain C scores, reflecting cognitive function, correlated with MMSE scores. Our results also confirmed the construct validity and intrarater reliability of the ABC-DS.

Clinical professionals in Japan often use assessments that can be administered in a short time, such as the MMSE and the HDS-R; however, these tests only measure cognitive function. In contrast, the ABC-DS can evaluate a patient in three domains - ADL function, BPSD, and cognitive function - in approximately $10 \mathrm{~min}$. Therefore, we named it ABC-DS. Unlike with the ROSA, a similar tool, the ABC-DS protocol does not require the assessor to judge the severity of a patient's AD before assessment. Moreover, it contains illustrations, and it is accompanied by easy-to-understand instructions; specific training on how to administer it is not necessary. Another major advantage is the predictive ability of the total ABC-DS score with respect to the CDR. Although the sensitivity for discriminating subjects with CDR $0 / 0.5$ from those with CDR $1 / 2 / 3$ is lower (0.68) than the other, the ABC-DS is useful as an index for judging the change of treatment, because it can distinguish between mild $\mathrm{AD}$ and moderate or severe AD. Furthermore, in contrast to the CDR, the ABC-DS has the advantage that it can evaluate BPSD. If a patient with mild AD undergoing treatment with cholinesterase inhibitors exhibits gradual deterioration in symptoms, decisions on when to increase the dosage or add another medication are usually made based on the information obtained from the patient's caregiver or his/her MMSE or HDS-R score. By allowing healthcare professionals to screen for moderate or severe AD in a short time, the ABC-DS can facilitate making suitable adjustments to a patient's drug regimen at each stage of the disease. Furthermore, changes in symptoms and drug efficacy can be observed by both total and domain-level ABC-DS scores. Healthcare providers can obtain detailed information about patients' symptoms by observing changes in domain-level scores. Observing changes in domain-level scores can help healthcare providers in dealing with not only cognitive function and ADL but also BPSD, as well as in giving detailed information to caregivers.

Most of the standard dementia rating scales currently in use are only assessed in terms of a total score (i.e., a simple sum). When factor analysis supports a one-factor solution for a scale, and its Cronbach $\alpha$ and $\omega$ reliability values are very close to 1.0, it may be fair to regard it as one-dimensional and use the total score for measuring that single trait. However, when factor analysis demonstrates the validity of grouping question items into multiple domains, those domains should be regarded as capturing different qualities. Representing different qualities using a single, total score may be mathematically inappropriate. Most standard rating scales used in clinical practice today adopt the single-factor position. In some cases, however, researchers did not even perform factor analysis during the development of their instrument.

The present study has some limitations and future challenges. First, because the ABC-DS was developed for the Japanese, reexamination of its reliability and validity will be necessary when adapting it to areas with different cultural backgrounds. In particular, it is necessary to study whether the same illustrations can be used as anchor points in regions with different cultural situations. Second, the ABC-DS has been developed for AD patients, and its validity for assessing other types of dementia has not been evaluated. Third, this study did not enroll any cognitively normal participants, because the ABC-DS was developed for use on patients who need treatment. We did not evaluate the possibility of discrimination between normal and MCI subjects or normal and AD subjects. Fourth, this study did not examine situations wherein the caregiver being interviewed changes between observation points (e.g., a patient's spouse being interviewed in the first assessment, and his/her child being interviewed in the next). Fifth, since new computerized adaptive testing of cognitive abilities, such as the NIH 
Toolbox [19], has been developed lately, we aim to compare the ABC-DS with such new scales in the future. Finally, the responsiveness of the ABC-DS to drug treatment should also be examined, considering that the responsiveness of the ROSA to drug treatment has already been investigated [20].

In conclusion, despite these limitations, the ABC-DS is effective in assessing symptoms and the severity of AD over time. Evaluators can calculate the score quickly and use it to facilitate decision-making about starting treatments or changing prescriptions.

\section{Acknowledgments}

We thank Mr. Hiromi Tsukada for the preparation of the data sets and performing part of the analyses, and Mr. Motohiro Sakamine for his substantial contribution as a project manager. We would also like to thank Prof. Masanori Fukushima for reviewing the manuscript.

The following doctors in the ABC Dementia Scale Study Group provided the data: Dr. Toshiyuki Watabe (Watabe Clinic), Dr. Akinori Ueki (Ueki Dementia and Geriatric Psychiatry Clinic), Dr. Hideki Yamamoto (Honmachi Clinic), Dr. Kitamura Yuri (Nanohana Clinic), Dr. Naruhiko Maki (MAKI HOSPITAL), Dr. Nobuya Kawabata (Shinwakai, Yachiyo Hospital), Dr. Hirotake Uchikado (Shonan Inaho Clinic), Dr. Naoto Kobayashi (Azuma Street Clinic), Dr. Chika Nishimura (Kurumi Clinic), Dr. Chiaki Kudoh (KUDOH CHIAKI Clinic for Neurosurgery and Neurology), Dr. Tomotake Otsuka (Nishikagawa Hospital), Dr. Masashi Takita (Takita Memory Mental Clinic), Dr. Kazuyoshi Harada (HARADA CLINIC), Dr. Hideki Ishizu (Zikei Hospital), Dr. Yumi Umeda-Kameyama (Taito Municipal Taito Hospital), Dr. Takashi Ueda (Medical Corporation Koujinkai, Ueda Neurosurgical Clinic), Assoc. Prof. Norifumi Tsuno (Kagawa University Hospital), Dr. Mitsuhiro Tsujihata (Nagasaki Kita Hospital), Dr. Kiyoshi Fujita (Okehazama Hospital Fujita Kokoro Care Center), Dr. Aoi Yoshiiwa (Takada Chuo Hospital), Dr. Masato Yamashita (Yamashita Clinic, Neurosurgery and Rehabilitation), and Prof. Masahiro Akishita (The University of Tokyo Hospital).

The present study is an academically initiated study financed by an independent grant from Daiichi Sankyo Co., Ltd. The company did not have any role in the study design, data collection, statistical analyses, or writing of the manuscript.

We would like to thank Editage (www.editage.jp) for English language editing.

\section{Statement of Ethics}

The study was conducted in accordance with the Declaration of Helsinki, following the approval from the ethics committee of the Kagawa University School of Medicine. Informed consent was obtained in writing from each patient or their legal guardian, as well as from their caregiver, before participation. The research was conducted by the Japanese Ministry of Health, Labour and Welfare's Ethical Guidelines for Medical Research Involving Human Subjects.

\section{Disclosure Statement}

In the past 5 years, T.M., Y.U.-K., K.W.-I., C.K., H.U., M.Y., T.W., C.N., N.T., T.U., M.A., and Y.N. have received honoraria from Daiichi Sankyo Co., Ltd. K.W.-I., M.A., and Kagawa University have received research funding from the company. 


\section{References}

1 Folstein MF, Folstein SE, McHugh PR: "Mini-mental state." A practical method for grading the cognitive state of patients for the clinician. J Psychiatr Res 1975;12:189-198.

2 Kato S, Shimogaki H, Onodera A, Ueda H, Oikawa K: Development of the revised version of Hasegawa's Dementia Scale (HDS-R) (in Japanese). Jpn J Geriatr Psychiatry 1991;2:1339-1347.

-3 Kim KW, Lee DY, Jhoo JH, Youn JC, Suh YJ, Jun YH, Seo EH, Woo JI: Diagnostic accuracy of Mini-Mental Status Examination and Revised Hasegawa Dementia Scale for Alzheimer's disease. Dement Geriatr Cogn Disord 2005;19:324-330.

4 Cummings JL, Mega M, Gray K, Rosenberg-Thompson S, Carusi DA, Gornbein J: The Neuropsychiatric Inventory: comprehensive assessment of psychopathology in dementia. Neurology 1994;44:2308-2314.

-5 Gélinas I, Gauthier L, McIntyre M, Gauthier S: Development of a functional measure for persons with Alzheimer's disease: the Disability Assessment for Dementia. Am J Occup Ther 1999;53:471-481.

6 Robert P, Ferris S, Gauthier S, Ihl R, Winblad B, Tennigkeit F: Review of Alzheimer's disease scales: is there a need for a new multi-domain scale for therapy evaluation in medical practice? Alzheimers Res Ther 2010;2: 24.

7 Morris JC: The Clinical Dementia Rating (CDR): current version and scoring rules. Neurology 1993;43:24122414.

8 Holthoff VA, Ferris S, Ihl R, Robert P, Winblad B, Gauthier S, Sternberg K, Tennigkeit F: Validation of the relevant outcome scale for Alzheimer's disease: a novel multidomain assessment for daily medical practice. Alzheimers Res Ther 2011;3:27.

9 American Psychiatric Association: Diagnostic and Statistical Manual of Mental Disorders, ed 4, text revision. Washington, American Psychiatric Association, 2000.

-10 McKhann GM, Knopman DS, Chertkow H, Hyman BT, Jack CR Jr, Kawas CH, Klunk WE, Koroshetz WJ, Manly JJ, Mayeux R, Mohs RC, Morris JC, Rossor MN, Scheltens P, Carrillo MC, Thies B, Weintraub S, Phelps CH: The diagnosis of dementia due to Alzheimer's disease: recommendations from the National Institute on AgingAlzheimer's Association workgroups on diagnostic guidelines for Alzheimer's disease. Alzheimers Dement 2011;7:263-269.

11 McKhann G, Drachman D, Folstein M, Katzman R, Price D, Stadlan EM: Clinical diagnosis of Alzheimer's disease: report of the NINCDS-ADRDA Work Group under the auspices of Department of Health and Human Services Task Force on Alzheimer's Disease. Neurology 1984;34:939-944.

-12 Albert MS, DeKosky ST, Dickson D, Dubois B, Feldman HH, Fox NC, Gamst A, Holtzman DM, Jagust WJ, Petersen RC, Snyder PJ, Carrillo MC, Thies B, Phelps CH: The diagnosis of mild cognitive impairment due to Alzheimer's disease: recommendations from the National Institute on Aging-Alzheimer's Association workgroups on diagnostic guidelines for Alzheimer's disease. Alzheimers Dement 2011;7:270-279.

13 Reise SP, Revicki DA: Handbook of Item Response Theory Modeling: Applications to Typical Performance Assessment, ed 1. New York, Routledge, 2014.

14 Mungas D, Reed BR: Application of item response theory for development of a global functioning measure of dementia with linear measurement properties. Stat Med 2000;19:1631-1644.

15 Wouters H, Zwinderman AH, van Gool WA, Schmand B, Lindeboom R: Adaptive cognitive testing in dementia. Int J Methods Psychiatr Res 2009;18:118-127.

16 Lindeboom R, Schmand B, Holman R, de Haan RJ, Vermeulen M: Improved brief assessment of cognition in aging and dementia. Neurology 2004;63:543-546.

17 Baker FB, Kim SH: The Basics of Item Response Theory Using R, ed 1. London, Springer, 2017.

18 Rizopoulos D: Itm: an R package for latent variable modeling. J Stat Softw 2006;17.

-19 Weintraub S, Dikmen SS, Heaton RK, Tulsky DS, Zelazo PD, Bauer PJ, Carlozzi NE, Slotkin J, Blitz D, WallnerAllen K, Fox NA, Beaumont JL, Mungas D, Nowinski CJ, Richler J, Deocampo JA, Anderson JE, Manly JJ, Borosh B, Havlik R, Conway K, Edwards E, Freund L, King JW, Moy C, Witt E, Gershon RC: Cognition assessment using the NIH Toolbox. Neurology 2013;80(suppl 3):S54-S64.

20 Holthoff V, Ferris S, Gauthier S, Ihl R, Robert P, Winblad B, Sternberg K, Tennigkeit F; ROSA Study Group: Memantine effects measured with the Relevant Outcome Scale for Alzheimer's Disease in an open-label, single-arm, multicenter clinical study. Int J Geriatr Psychiatry 2013;28:164-172. 